\title{
Modeling Microscopic Car-Following Strategy of Mixed Traffic to Identify Optimal Platoon Configurations for Multiobjective Decision-Making
}

\author{
Mudasser Seraj $\mathbb{D}^{1}{ }^{1}$ Jiangchen Li $\mathbb{D},{ }^{1}$ and Zhijun Qiu $\mathbb{D}^{1,2}$ \\ ${ }^{1}$ Department of Civil and Environmental Engineering, University of Alberta, Edmonton, Alberta, Canada \\ ${ }^{2}$ Intelligent Transportation System Research Center, Wuhan University of Technology, Wuhan, Hubei, China \\ Correspondence should be addressed to Zhijun Qiu; zhijunqiu@ualberta.ca
}

Received 31 May 2018; Revised 16 August 2018; Accepted 10 September 2018; Published 27 September 2018

Guest Editor: Guoyuan Wu

Copyright (C) 2018 Mudasser Seraj et al. This is an open access article distributed under the Creative Commons Attribution License, which permits unrestricted use, distribution, and reproduction in any medium, provided the original work is properly cited.

\begin{abstract}
Microscopic detail of complex vehicle interactions in mixed traffic, involving manual driving system (MDS) and automated driving system (ADS), is imperative in determining the extent of response by ADS vehicles in the connected automated vehicle (CAV) environment. In this context, this paper proposes a naïve microscopic car-following strategy for a mixed traffic stream in CAV settings and specified shifts in traffic mobility, safety, and environmental features. Additionally, this study explores the influences of platoon properties (i.e., intra-platoon headway, inter-platoon headway, and maximum platoon length) on traffic stream characteristics. Different combinations of MDS and ADS vehicles are simulated in order to understand the variations of improvements induced by ADS vehicles in a traffic stream. Simulation results reveal that grouping ADS vehicles at the front of traffic stream to apply Cooperative Adaptive Cruise Control (CACC) based car-following model will generate maximum mobility benefits for upstream vehicles. Both mobility and environmental improvements can be realized by forming long, closely spaced ADS vehicles at the cost of reduced safety. To achieve balanced mobility, safety, and environmental advantages from mixed traffic environment, dynamically optimized platoon configurations should be determined at varying traffic conditions and ADS market penetrations.
\end{abstract}

\section{Introduction}

Vehicles with diverse levels of integrated connectivity and automated control systems are considered to be pushing a technological leap towards diminished trip delay, fuel efficiency, reduced emission, and enhanced safety of road traffic. Although a purely automated vehicle-based traffic stream could take decades to become a reality, introducing and gradually increasing market shares of automated driving system-based vehicles in traffic streams would enable us to perceive and harness the potential gains from these technologies. Varied perceptions of mixed traffic streams and their collaborative motion dynamics hindered both researchers and practitioners from progressing with these technologies. Furthermore, the ideal compositions of automated vehicles in mixed traffic conditions remain unfamiliar to most. In response to these problems, this study proposes a simple yet effective car-following strategy for mixed traffic stream and measures the resulting impact on mobility, safety, and the environment. Additionally, the car-following strategy involved platoon development in a connected automated vehicle (CAV) environment and the study explores various platoon configurations to determine platoon parameters at different traffic states to obtain utmost benefits.

Numerous studies have been conducted by acclaimed researchers and practitioners to interpret the complex dynamics of combined traffic movements [1-6]. While these studies transcended in conceiving the levels of impact of automated driving technologies through simplified to complex macroscopic and mesoscopic modeling, the motivation of the present was shaped by the need of modeling microscopic car-following behavior in heterogeneous traffic in order to study macroscopic consequences from mobility, safety, and environmental perspectives. With that intention, this study 
gives insights into a wide variation of distinct forms of impact while simulating automated-control-enabled vehicles on ideal locations and distributions along traffic stream. These insights into mixed traffic movements and platoon characteristics will motivate researchers to consider other unattended aspects of mixed traffic dynamics (e.g., lanechanging, gap acceptance, and merging) in order to rectify perceived benefits. Similarly, traffic operational authorities can take these lessons into account to impose different control strategies (e.g., dynamic aggregated controls for manually driven vehicles, dynamic personalized controls on connected vehicles) on traffic to attain maximum improvements with regard to reduced travel time, collision rates, greenhouse gas emissions, etc.

The rest of the paper is organized as follows: The next section summarizes the existing literature on car-following models and strategies for mixed traffic and also touches on studies that identify the different form of impact that automated vehicles have on traffic. The proposed car-following strategy is described in the following section. The description of simulation procedures and the discussion on obtained results are covered, respectively, in two subsequent sections. The following section proposed an approach to obtain dynamic optimal platoon configuration for specific traffic state. The last section provides the synopsis of findings of the study and also gives recommendations for future research.

\section{Literature Review}

As the primary aim of this study relates to car-following strategy for mixed traffic environment, the literature related to car-following models for both forms (i.e., manual, automated) of driving system is explored here. Numerous microscopic car-following models have been proposed to imitate driving pattern of manual driving system [7-12]. Among the proposed stimulus-response-based car-following models, the intelligent driver model (IDM) is widely used in literature to depict manual driving dynamics. The ability of this model to define numerous microscopic (e.g., desired velocity and acceleration/deceleration limits) and macroscopic (e.g., capacity, capacity drop, and fundamental diagram) phenomena made it the prevalent model. On the other hand, due to rapid growth of CAV technology, the longitudinal control models for automated vehicles were also examined by researchers [13-18]. These studies provide us with structures to work on car-following strategy in mixed traffic environment and identify the extents of potential paradigm shifts.

A clear distinction of the driving system is dictated by the operational authority. While the manual driving system (MDS) represents driving systems controlled by humans, the motion dynamics of vehicles with the automated driving system (ADS) are mandated by distinct levels of automation. ADS vehicles' longitudinal movements are commonly portrayed with adaptive cruise control (ACC) and cooperative adaptive cruise control (CACC). Many studies have analyzed the contributions of longitudinal control system of ADS vehicles on traffic mobility [19-28]. While mobility was the main focus of these studies, the impact of traffic movement from safety and environmental perspective was often ignored.
Yeo et al. [29] proposed an integrated car-following and lane changing model to perform microsimulation of oversaturated freeway traffic. The proposed algorithm considered complex dynamic interactions at a microscopic level to replicate vehicle movements. However, the aptitude of this model to capture possible consequences was not tested. Wang et al. [30] proposed a car-following control for autonomous vehicle and identified the impact, focusing mainly on traffic flow characteristics. Liberis et al. [31] took a macroscopic approach to identify traffic mobility parameters in a heterogeneous traffic environment. The authors used the market penetration rate of connected vehicles to estimate traffic states. Moreover, other researchers studied the impact of introducing ADS based vehicles with conventional vehicles [32-36] on flow and mobility. Reviews of these studies provide us with the opportunity to constructively examine the contributions of earlier studies, identify the necessities to improve current knowledge, and uncover the latent insights to progress promptly with CA technology.

While the mobility attributes of traffic flow were widely discussed in many studies, the safety and environmental aspects, which are equally if not more important, were relatively unexplored by a majority of the studies. The impact of automated vehicles on both safety and mobility was discussed by Fernandes and Nunes [37]. They studied platooning of ADS vehicles with different communication schemes at various flow rates to improve roadway capacity. Several studies assessed the safety aspects of CAV based traffic. According to the National Highway Traffic Safety Administration (NHTSA), a complete adaptation of CAV based traffic movements would annually prevent 439,000-615,000 crashes [38]. Li et al. [39] evaluated the impact of CACC control on reducing rear-end collisions on freeways. The study shows a reduction in safety improvements with increasing market share of ADS vehicles. Rahman and Abdel-Aty [40] compared potential improvement in longitudinal safety due to varying market penetration of connected vehicles. According to the analysis presented, the managed-lane CAC control outperformed multilane control with regard to traffic safety. The report of Zabat et al. [41] stated that the presence of boundary layer along closely spaced vehicles would reduce aerodynamic drag, resulting in reduced fuel consumption and less emission. Platoon-wide environmentfriendly CACC system was studied by Wang et al. [42] and their objective assessment attained 2\% fuel saving with $17 \%$ emission reductions. Mamouei et al [43] argued that fueleconomy based ACC control model would not lead to highly conservative driving dynamics of traffic.

Although the reviewed studies had remarkable contributions that helped to clarify the roles and influences of ADS vehicles in traffic, the inadequacy of multiobjective decision-making approach to address ADS vehicles' potential has influenced this research further to investigate the complex interdependencies of mixed traffic. This research seeks to contribute on three research gaps identified from the literature. These gaps are (i) the significance of ADS vehicles' position and distributions along traffic stream, (ii) the variations of traffic flow attributes (i.e., mobility, safety, and environmental) resulting from structural changes of 


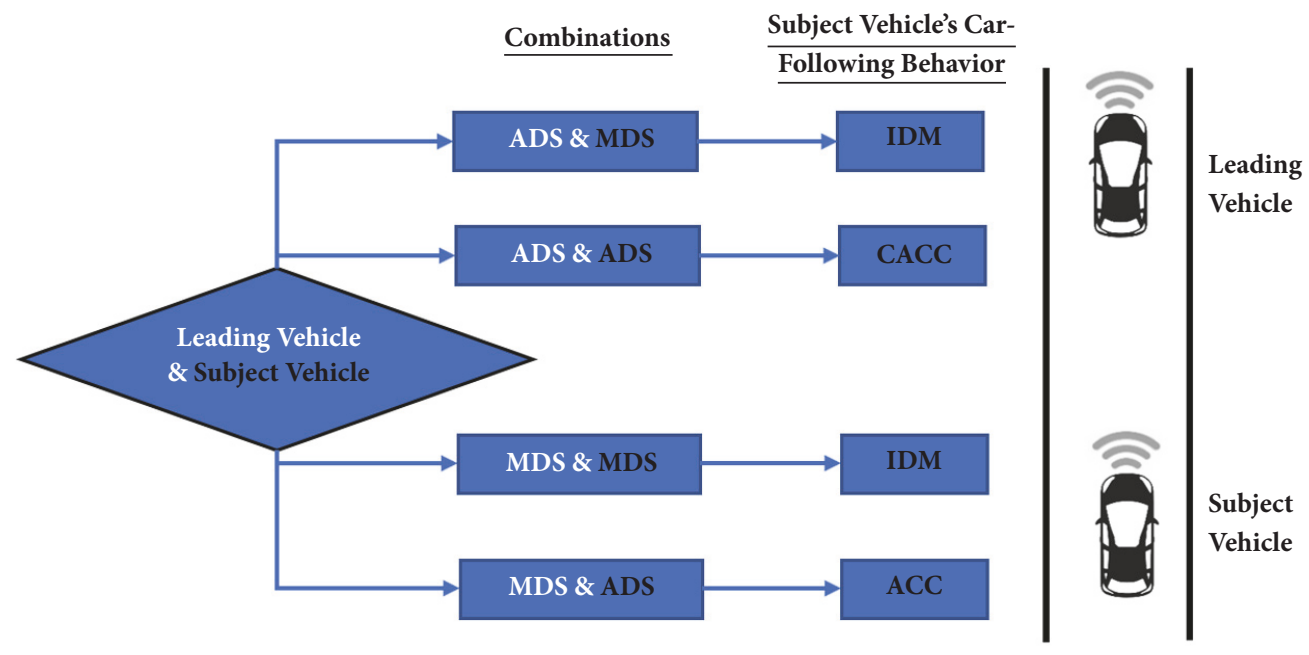

FIGURE 1: Proposed car-following strategy for mixed traffic.

CACC platoons, and (iii) adjusting platoon configurations dynamically to obtain balanced benefits from considered traffic attributes.

\section{Proposed Car-Following Strategy for Individual Vehicle}

Interactions and behaviors of vehicles at microscopic levels have macroscopic implications. Factors like maximum accelerations, comfortable decelerations, preferred time headways, etc. are directly linked to traffic mobility, safety, and environmental aspects. Car-following models provide individual vehicles' acceleration from dynamic interactions with adjacent vehicles, control constraints to generate velocity, and position to determine vehicle trajectory. The car-following models of vehicles in mixed traffic were schemed here to simulate real-traffic movements. As mentioned earlier, the existence of two types of vehicle driving system was considered for combined traffic. The proposed driving strategy identified all potential combinations of leading vehicle and subject vehicle based on driving systems to determine suitable car-following models.

The proposed car-following mechanism presumed that MDS vehicles would maintain conventional car-following behavior irrespective of the leading vehicle's driving system [Figure 1]. In this regard, intelligent driver model (IDM) [10] was chosen to represent manual drivers' car-following behavior. Extensive applications of this model across different studies developed this model as a perfect example to simulate MDS vehicles' car-following behavior. An enhanced version of traditional IDM was used to determine a realistic longitudinal control decision of MDS vehicles (see (1)). Discretized kinematic equations were used for all vehicles irrespective of the driving system to determine vehicle's velocity and position (see (2) and (3)).

$$
\dot{v}(t+\Delta t)=a\left[1-\left(\frac{v(t)}{v_{0}}\right)^{4}\right.
$$

$$
\left.-\left(\frac{s_{0}+\max [0, v(t) \times T+(v(t) \times \Delta v(t)) / 2 \sqrt{a b}]}{s}\right)^{2}\right]
$$

$$
\begin{aligned}
& v(t+\Delta t)=v(t)+a(t) \times \Delta t \\
& p(t+\Delta t)=p(t)+v(t) \times \Delta t+\frac{1}{2} a(t) \times \Delta t^{2}
\end{aligned}
$$

where $\dot{v}$ is acceleration of vehicle $\left(\mathrm{m} / \mathrm{s}^{2}\right), \mathrm{v}$ is velocity $(\mathrm{m} / \mathrm{s}), p$ is vehicle position $(\mathrm{m}), a$ is maximum acceleration (set as $3 \mathrm{~m} / \mathrm{s}^{2}$ ), $v_{0}$ is desired velocity $(35 \mathrm{~m} / \mathrm{s}), s_{0}$ is leading gap at jam density $(5 \mathrm{~m}), b$ is desirable deceleration $\left(-3 \mathrm{~m} / \mathrm{s}^{2}\right), \Delta v$ is velocity difference with leading vehicle, and $T$ is preferred time headway $(2.5 \mathrm{sec})$.

To demonstrate the car-following mechanism of ADS vehicles, both ACC and CACC based car-following were implemented. A MDS based leading vehicle would prompt ADS vehicle to follow ACC with relatively high preferred time headway. Provided that the leading vehicle had ADS, the subject vehicle would choose CACC based car-following. Whether the subject vehicle would join the CACC platoon depends on the leading vehicle's platoon ID. Platoon ID is an identification number assigned to an ADS vehicle that represents its order of position in the platoon. If an ADS vehicle is a part of a platoon, it will have a fixed platoon ID; otherwise its platoon ID will contain a platoon ID $=0$ (zero). While travelling through roads, the built-in communication technology of ADS vehicles would enable them to identify the leading vehicles driving system as well as platoon ID. If the platoon ID of the leading vehicle was equal to the maximum platoon length, then the subject vehicle would form a new platoon by maintaining inter-platoon headway and as a leader of the new platoon. In addition, if the leading vehicle's platoon ID was lower than maximum platoon length, the subject vehicle would join the platoon by maintaining intra-platoon headway. We adopted the ACC and CACC car-following models developed in [17]. The accelerations of the subject 
vehicle were determined with respect to relative position and velocity. The following equation was used to determine the acceleration of the subject vehicle:

$$
\dot{v}(t+\Delta t)=k_{1}\left(\Delta p(t)-v(t) \times T-s_{0}\right)+k_{2} \Delta v(t)
$$

where $k_{1}, k_{2}$ are control constants for relative distance and speed, respectively $\left(k_{1}, k_{2}>0\right)$ and $\Delta p(t)$ is position difference with leading vehicle. The stability of the proposed ACC system was proved in [17]. Suitable $k_{1}, k_{2}$ values were chosen according to [17] to implement realistic simulation accounting for the sensitivity of these factors. Similar approach of dual consensus was taken by Wang et al. [18] where both position and velocity consensus were considered to determine acceleration/deceleration decision. While both ACC and CACC car-following models used (4) to determine acceleration values for ADS vehicles, higher preferred time headways ( $T=1.5 \mathrm{sec}$ ) distinguish ACC mode with CACC mode $(T \leq 1.0 \mathrm{sec})$.

\section{Simulation Procedures}

A microscopic simulation structure was built on MATLAB to replicate vehicles' motion on a two-lane directional highway. The simulation environment was grounded on numerical analysis-based car-following behavior. All previously mentioned motion dynamic equations were coded to follow proposed car-following strategy. A stream of 20 vehicles following a controlled leading vehicle was simulated for numerous scenarios. The time headways between the vehicles in traffic stream were manipulated to simulate distinct traffic flow rates. In the simulation environment, the acceleration of the first vehicle was controlled consciously to generate multiple shockwaves and to observe the reaction of the vehicles behind it. Each simulation ran for 1000 time steps and 20 times for each scenario. The preferred time headway (T) for MDS vehicles was considered as a normally distributed variable with mean value of $2.5 \mathrm{sec}$ and standard deviation of $0.5 \mathrm{sec}$. Multiple runs for each scenario were executed to ensure that the obtained outcome was free from anomaly. The average values of 20 runs were listed for analysis. In the beginning of the simulation, the first vehicle was travelling at $25 \mathrm{~m} / \mathrm{s}$ for 210 time steps and then accelerated at $0.167 \mathrm{~m} / \mathrm{s}^{3}$ rate for 60 time steps followed by steady state (acceleration/deceleration rate $=0 \mathrm{~m} / \mathrm{s}^{3}$, velocity $=35 \mathrm{~m} / \mathrm{s}$ ) for 120 time steps. Finally, the controlled vehicle at front decelerated again at $0.167 \mathrm{~m} / \mathrm{s}^{3}$ rate for 60 time steps to regain $25 \mathrm{~m} / \mathrm{s}$ velocity and moved with constant velocity for the remaining time steps. The maximum velocity was set to $35 \mathrm{~m} / \mathrm{s}$. The combinations generated from the following variables sets were simulated to represent various traffic states encountered in roadways as well as to identify the variations on improvements obtained by introducing the ADS vehicles in the connected automated vehicle (CAV) environment:

(a) Initial flow rate (veh/hr): (i) 1400, (ii) 1800, (iii) 2400

(b) ADS market share (\%): (i) 25, (ii) 50, (iii) 75

(c) Maximum platoon length (vehicle): (i) 3, (ii) 4, (iii) 5 , (iv) 6 (d) Inter-platoon headway (sec): (i) 2, (ii) 4, (iii) 6, (iv) 8

(e) Intra-platoon headway (sec): (i) 0.5 , (ii) 0.75 , (iii) 1.0, (iv) 1.25

The variables sets were restricted by the above values to limit the analysis and discussions within manageable ranges while covering a wide range of variations in traffic conditions. Platoon parameters (i.e., maximum platoon length, interplatoon headway, and intra-platoon headway) were varied within reasonable ranges to identify observable trends. Two distinct driving systems were simulated by assigning specific values of driving system ( 0 for MDS, 1 for ADS). The driving system values assigned for vehicles were used to implement the proposed car-following strategy on the CAV environment. Assigned driving system values were also useful to adopt proper sets of motion dynamic equations.

\section{Analysis, Results, and Findings}

5.1. Impact of ADS Vehicles Location and Distribution. Before analyzing the mobility, safety, and environmental aspects of ADS vehicles on traffic, the influences of ADS vehicles location and distribution in traffic stream were explored. It was hypothesized that the positions of ADS vehicles in traffic stream dictated their impacts on the remaining vehicles. To prove this hypothesis, the proposed car-following strategy was simulated by allotting ADS vehicles at diverse combinations of positions with gradually increasing the initial flow rate and ADS market share. To clearly comprehend the significance of vehicle position more clearly and to reduce the intricacy of comprehension, only two features were analyzed: acceleration fluctuation of MDS vehicle in the vehicle group and variations of maximum traffic flow at varying traffic state. Since numerous combinations of ADS vehicles' distribution are viable at different penetration rates of ADS vehicles, only a handful of combinations were selected to cover most possible variations.

Initially, these distributions were generated by placing ADS vehicles as far apart as possible (--\% Comb-1) in the vehicle stream while maintaining target ADS market share. Gradually, ADS vehicles were grouped together in different combinations. The purpose of placing ADS vehicles in such an order was to visualize and measure the impact of ADS vehicles location and distribution along the vehicle stream. The combinations are listed in Table 1. The first column of the table shows percentages of ADS vehicles in the traffic stream. The numbers in second column of Table 1 identify the position ID of ADS vehicles in the traffic stream. Other vehicles, except the positions mentioned in the table, were MDS vehicles. The last column of the table provides distinct combination name of each distribution of ADS vehicles. These combinations were simulated on developed simulation environment by virtually placing ADS vehicles in the mentioned position IDs of the vehicle stream and by following proposed car-following strategy for mixed traffic. The listed combinations in Table 1 were assumed to represent varying ranges of $A D S$ vehicles distribution on vehicle group. Analyzing these sets of vehicle location and distribution 
TABLE 1: List of ADS vehicle combinations simulated for different market penetrations.

\begin{tabular}{|c|c|c|}
\hline ADS Market Share & Distribution of ADS vehicles (position) & Combination Name \\
\hline \multirow{6}{*}{$25 \%$} & $4,8,12,16,20$ & $25 \%$ Comb-1 \\
\hline & $4,5,10,11,16$ & $25 \%$ Comb-2 \\
\hline & $5,6,7,13,14$ & $25 \%$ Comb-3 \\
\hline & $9,10,11,1217$ & $25 \%$ Comb- 4 \\
\hline & $2,3,4,5,6$ & $25 \%$ Comb-5 \\
\hline & $16,17,18,19,20$ & $25 \%$ Comb- 6 \\
\hline \multirow{5}{*}{$50 \%$} & $2,4,6,8,10,12,14,16,18,20$ & $50 \%$ Comb -1 \\
\hline & $2,3,6,7,10,11,14,15,18,19$ & $50 \%$ Comb -2 \\
\hline & $2,3,4,8,9,10,14,15,16,20$ & $50 \%$ Comb -3 \\
\hline & $2,3,4,5,10,11,12,13,18,19$ & $50 \%$ Comb -4 \\
\hline & $2,3,4,5,6,7,8,9,10,11$ & $50 \%$ Comb -5 \\
\hline \multirow{3}{*}{$75 \%$} & $2,3,4,6,7,8,10,11,12,14,15,16,18,19,20$ & $75 \%$ Comb-1 \\
\hline & $2,3,4,5,6,9,10,11,12,13,16,17,18,19,20$ & $75 \%$ Comb- 2 \\
\hline & $2,3,4,5,6,9,10,11,12,13,16,17,18,19,20$ & 75\% Comb-3 \\
\hline
\end{tabular}

provided the opportunity to shed light on resulting impacts due to ADS vehicles' position on traffic stream.

From the analysis, the simulation outcomes of the initial flow rate of $1800 \mathrm{veh} / \mathrm{hr}$ with different ADS market penetration are provided in Figure 2 to demonstrate the influences of ADS vehicles position and distribution along the stream from both microscopic and macroscopic perspective. Figure 2(a) represents the variations of maximum flow rates resulting from the proposed car-following strategy at listed combinations. Figure 2(b) shows the average coefficient of variations $(\mathrm{CoV})$ of acceleration of $\mathrm{MDS}$ vehicles in the simulated vehicle stream. Boxplots for a specific combination were plotted from the maximum flow rate and average $\mathrm{CoV}$ of acceleration data of simulated scenarios with varying platoon parameters, as listed before. Macroscopic analysis on maximum flow rates at different ADS vehicle shares (Figure 2(a)) identified the pattern of gradual increment with increasing ADS shares in the traffic. Observations of different combinations revealed that combinations with scattered ADS vehicles lead to lower maximum flow rates in comparison to combinations with grouped ADS vehicles. Additionally, grouping $\mathrm{ADS}$ vehicles at the front of the vehicle stream (i.e., 25\% Comb-6, 50\% Comb-5, and 75\% Comb-3) resulted in $6.7-11.5 \%$ higher maximum flow rates in comparison to the scattered distribution of ADS vehicles (i.e., 25\% Comb1, 50\% Comb-1, and 75\% Comb-1). Analysis on microscopic characteristics of MDS vehicles was undertaken by measuring the average $\mathrm{CoV}$ of acceleration at different market shares and combinations of ADS vehicles. The resulting analysis showed a gradual decreasing $\mathrm{CoV}$ of acceleration with increasing shares of ADS vehicles. Similar to macroscopic analysis, the maximum amount of decrease in $\mathrm{CoV}$ (1.69-6.63\%) was observed from combinations with ADS vehicles at the front of the traffic stream grouped together. Specific analysis on maximum platoon length's influence on acceleration fluctuations of MDS vehicles revealed that increasing maximum platoon length reduced the average coefficient of variation of acceleration for ADS vehicles. Similar analysis on the other two platoon parameters (i.e., inter-platoon headway and intra-platoon headway) demonstrated a reciprocal relation with acceleration fluctuations (increasing inter- and intraplatoon headway increased the average $\mathrm{CoV}$ of acceleration).

The analysis of the remaining initial flow rates and ADS market share revealed that creating platoons of ADS vehicles by positioning them at the front of traffic stream would be beneficial to the rest of vehicles in the traffic stream. Furthermore, increasing market shares of ADS vehicles could gradually reduce the acceleration fluctuation of MDS vehicles. Finally, increasing the flow rates could inversely influence traffic flow improvements with a specific ADS location and distribution combination. The notion of traffic flow improvements guided the authors in this study to explore mobility improvement potentials of the proposed carfollowing strategy by placing ADS vehicles at ideal positions along the traffic stream.

5.2. Impact on Mobility. Since creating platoons of ADS vehicles was found to be the most effective way of acquiring associated benefits, influences of ADS vehicles on traffic mobility were examined with respect to three key variables of platooning: intra-platoon headway, inter-platoon headway, and maximum platoon length. Combinations of these three variables within listed sets were utilized to generate various platoon scenarios for simulation and analysis. The impact of these platoon structures on mobility was measured and compared with the help of two parameters: Average Travel Time (ATT) (see (5)) and Average Travel Distance (ATD) (see (6)). Later, case scores were computed by providing equal weights to ATT and ATD (see (7)). Different cases of platoon configurations were simulated and evaluated through case scores. Higher dispersion from base-case (0\% ADS share) scores indicated higher mobility improvements. The objective of this analysis was to identify the optimal platoon configuration to improve mobility by increasing ATD and reducing ATT. The following equations were used to identify the mobility gains.

$$
A T T=\frac{\sum_{j=1}^{J} A T T_{j}}{J}=\sum_{i=1}^{I} \frac{\left(p_{i, j}-p_{i, j-1}\right)}{v_{i, j}}
$$



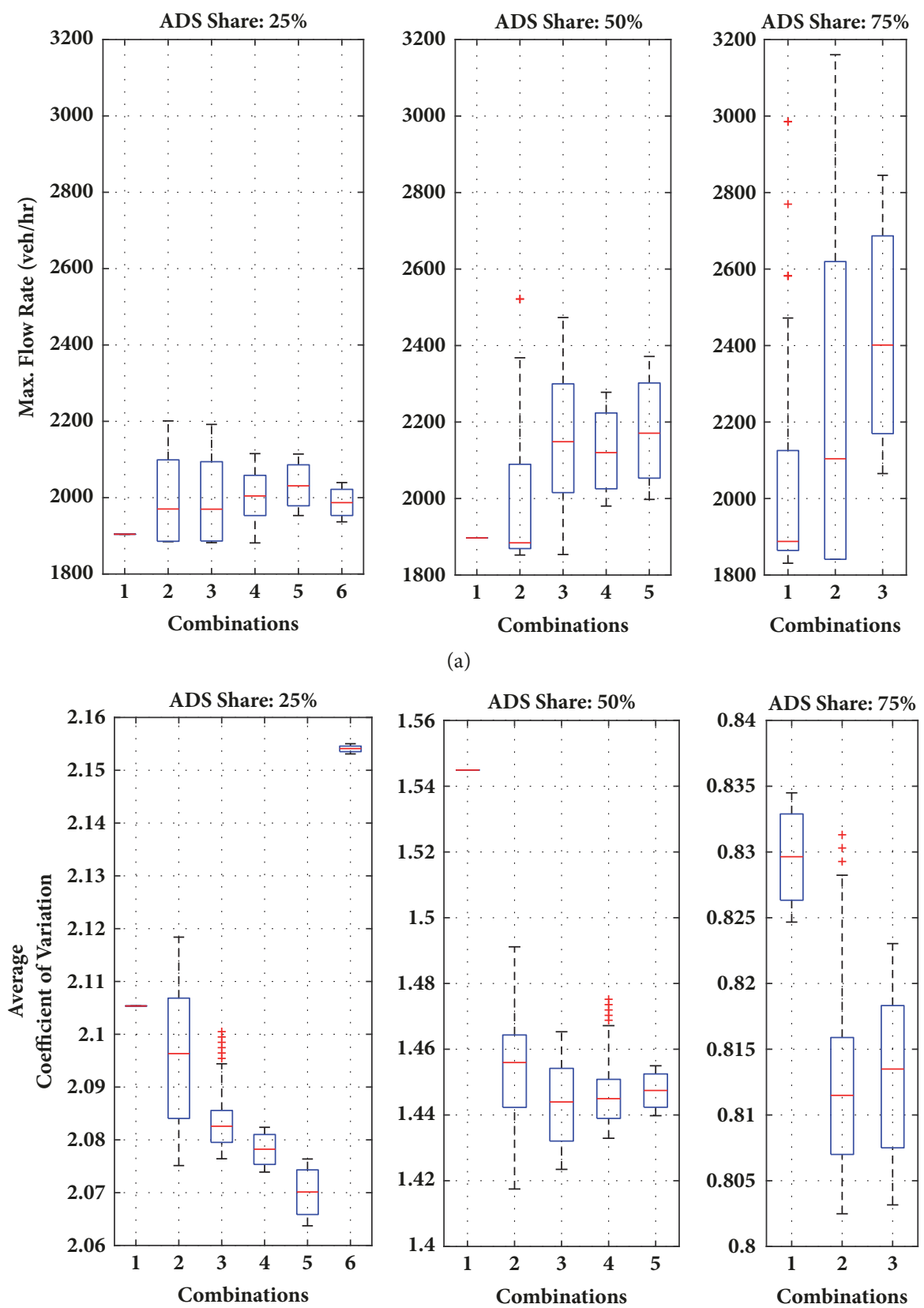

(b)

FIGURE 2: Influences of ADS vehicles' position on (a) maximum flow rate and (b) average coefficient of variation of accelerations.

$$
\begin{aligned}
A T D & =\frac{\sum_{J=1}^{J} A T D_{j}}{J}, \\
A T D_{j} & =\frac{\left(p_{I, j}-p_{1, j}\right)}{I} \\
\text { Score }_{\text {Case }} & =\frac{\sum_{J=1}^{J} v_{1, j}\left(A T D_{j, \text { Case }}\right)}{J}-\left(A T T_{\text {Case }}\right)
\end{aligned}
$$

Here, $i$ is vehicle index $(I=21), j$ is time index ( $J$ $=1000), v_{i, j}$ is velocity of vehicle $\mathrm{i}$ at time step $\mathrm{j}, p_{i, j}$ is position of vehicle $\mathrm{i}$ at time step $\mathrm{j}$, and Score $_{\text {SCase }}$ is score of case $\mathrm{k}$. Aforementioned (Section 4) platoon variables (i.e., maximum platoon length, inter-platoon headway, and intra-platoon headway) were explored to generate distinct platoon scenarios. The combinations of these parameter sets produced 64 distinct platoon configuration cases that were simulated for different traffic flows and ADS market shares to detect the capability of mobility improvements. Moreover, the limits of mobility improvements due to variation of platoon configurations were also revealed in this analysis. Obtained mobility improvements from base cases at different traffic states are presented in Figure 3(a). The three-quarter circles showed comparative mobility progresses at different flow 


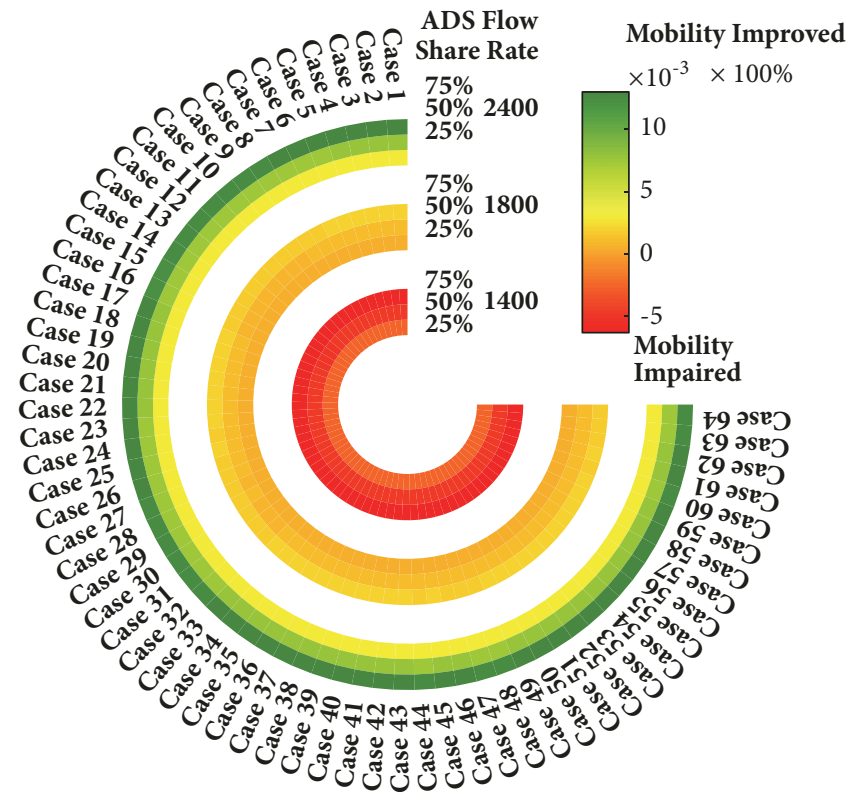

(a)

\begin{tabular}{|c|c|c|c|c|c|c|c|c|c|}
\hline Case & \begin{tabular}{|l|} 
Intra \\
Platoon \\
Headway
\end{tabular} & \begin{tabular}{|l} 
Inter \\
Platoon \\
Headway
\end{tabular} & $\begin{array}{l}\text { Max. } \\
\text { Platoon } \\
\text { Length }\end{array}$ & $\begin{array}{c}\text { Mobility } \\
\text { Improvement }\end{array}$ & Case & $\begin{array}{l}\text { Intra } \\
\text { Platoon } \\
\text { Headway }\end{array}$ & \begin{tabular}{|c} 
Inter \\
Platoon \\
Headway
\end{tabular} & $\begin{array}{l}\text { Max. } \\
\text { Platoon } \\
\text { Length }\end{array}$ & $\begin{array}{c}\text { Mobility } \\
\text { Improvemen }\end{array}$ \\
\hline 1 & 0.50 & 2 & 3 & $0.200 \%$ & 33 & 0.50 & 2 & 5 & $0.204 \%$ \\
\hline 2 & 0.75 & 2 & 3 & $0.196 \%$ & 34 & 0.75 & 2 & 5 & $0.200 \%$ \\
\hline 3 & 1.00 & 2 & 3 & $0.193 \%$ & 35 & 1.00 & 2 & 5 & $0.196 \%$ \\
\hline 4 & 1.25 & 2 & 3 & $0.190 \%$ & 36 & 1.25 & 2 & 5 & $0.192 \%$ \\
\hline 5 & 0.50 & 4 & 3 & $0.189 \%$ & 37 & 0.50 & 4 & 5 & $0.198 \%$ \\
\hline 6 & 0.75 & 4 & 3 & $0.186 \%$ & 38 & 0.75 & 4 & 5 & $0.194 \%$ \\
\hline 7 & 1.00 & 4 & 3 & $0.182 \%$ & 39 & 1.00 & 4 & 5 & $0.190 \%$ \\
\hline 8 & 1.25 & 4 & 3 & $0.179 \%$ & 40 & 1.25 & 4 & 5 & $0.186 \%$ \\
\hline 9 & 0.50 & 6 & 3 & $0.178 \%$ & 41 & 0.50 & 6 & 5 & $0.193 \%$ \\
\hline 10 & 0.75 & 6 & 3 & $0.175 \%$ & 42 & 0.75 & 6 & 5 & $0.189 \%$ \\
\hline 11 & 1.00 & 6 & 3 & $0.172 \%$ & 43 & 1.00 & 6 & 5 & $0.185 \%$ \\
\hline 12 & 1.25 & 6 & 3 & $0.168 \%$ & 44 & 1.25 & 6 & 5 & $0.181 \%$ \\
\hline 13 & 0.50 & 8 & 3 & $0.168 \%$ & 45 & 0.50 & 8 & 5 & $0.188 \%$ \\
\hline 14 & 0.75 & 8 & 3 & $0.164 \%$ & 46 & 0.75 & 8 & 5 & $0.184 \%$ \\
\hline 15 & 1.00 & 8 & 3 & $0.161 \%$ & 47 & 1.00 & 8 & 5 & $0.180 \%$ \\
\hline 16 & 1.25 & 8 & 3 & $0.158 \%$ & 48 & 1.25 & 8 & 5 & $0.176 \%$ \\
\hline 17 & 0.50 & 2 & 4 & $0.202 \%$ & 49 & 0.50 & 2 & 6 & $0.204 \%$ \\
\hline 18 & 0.75 & 2 & 4 & $0.198 \%$ & 50 & 0.75 & 2 & 6 & $0.200 \%$ \\
\hline 19 & 1.00 & 2 & 4 & $0.194 \%$ & 51 & 1.00 & 2 & 6 & $0.196 \%$ \\
\hline 20 & 1.25 & 2 & 4 & $0.191 \%$ & 52 & 1.25 & 2 & 6 & $0.192 \%$ \\
\hline 21 & 0.50 & 4 & 4 & $0.194 \%$ & 53 & 0.50 & 4 & 6 & $0.198 \%$ \\
\hline 22 & 0.75 & 4 & 4 & $0.190 \%$ & 54 & 0.75 & 4 & 6 & $0.194 \%$ \\
\hline 23 & 1.00 & 4 & 4 & $0.186 \%$ & 55 & 1.00 & 4 & 6 & $0.190 \%$ \\
\hline 24 & 1.25 & 4 & 4 & $0.183 \%$ & 56 & 1.25 & 4 & 6 & $0.186 \%$ \\
\hline 25 & 0.50 & 6 & 4 & $0.186 \%$ & 57 & 0.50 & 6 & 6 & $0.193 \%$ \\
\hline 26 & 0.75 & 6 & 4 & $0.182 \%$ & 58 & 0.75 & 6 & 6 & $0.189 \%$ \\
\hline 27 & 1.00 & 6 & 4 & $0.178 \%$ & 59 & 1.00 & 6 & 6 & $0.185 \%$ \\
\hline 28 & 1.25 & 6 & 4 & $0.175 \%$ & 60 & 1.25 & 6 & 6 & $0.181 \%$ \\
\hline 29 & 0.50 & 8 & 4 & $0.178 \%$ & 61 & 0.50 & 8 & 6 & $0.188 \%$ \\
\hline 30 & 0.75 & 8 & 4 & $0.174 \%$ & 62 & 0.75 & 8 & 6 & $0.184 \%$ \\
\hline 31 & 1.00 & 8 & 4 & $0.170 \%$ & 63 & 1.00 & 8 & 6 & $0.180 \%$ \\
\hline 32 & 1.25 & 8 & 4 & $0.167 \%$ & 64 & 1.25 & 8 & 6 & $0.176 \%$ \\
\hline
\end{tabular}

(b)

FIGURE 3: Variations of mobility benefits due to varying platoon configurations at (a) different flow rates and ADS shares and (b) specific flow rate (1800 veh/hr) and ADS share (75\%). 
rates and ADS market shares simulated for the analysis. The color bar in Figure 3(a) indicated the extent of generated mobility score improvements. Figure 3(b) reveals detailed analysis for a specific flow rate and ADS share. For clear understanding of the impact of platoon configurations at a specific traffic state, mobility improvements at initial flow rate of $1800 \mathrm{veh} / \mathrm{hr}$ and $75 \%$ ADS share are provided in Figure 3(b) as an example. As observed in Figure 3(b), sixty-four (64) separate platoon configurations are generated from listed parameter set (Section 4). Parameters for each case are listed in the table in Figure 3(b). The mobility improvement column was calculated by comparing the base case (flow rate $=1800 \mathrm{veh} / \mathrm{hr}$, ADS share $=0 \%$ ) with the corresponding cases and transforming the value into a percentage. Negative percentages indicate impaired mobility and positive percentages denote improved mobility resulting from a specific platoon configuration. When inspecting Figure 3(b), it was found that maximum mobility benefits (0.204\% improvement on case score) could be obtained from Case 33 (platoon configuration: intra-platoon headway = $0.50 \mathrm{sec}$, inter-platoon headway $=2 \mathrm{sec}$, and max. platoon length $=5$ ) and Case 49 (platoon configuration: intra-platoon headway $=0.50 \mathrm{sec}$, inter-platoon headway $=2 \mathrm{sec}$, and max. platoon length $=6$ ) for that specific traffic state. A declining trend of mobility gains was captured with increasing inter and intra-platoon headway. Additionally, increasing maximum platoon length parameter showed expansion with regard to mobility which came to a halt at maximum platoon length $=$ 5 .

An exploration of Figure 3(a) revealed that increasing ADS market could bring broader mobility enhancement at higher flow rates (yellow to green bands on $2400 \mathrm{veh} / \mathrm{hr}$ flow rate). Increased ADS share at low flow rates had a diminishing effect on mobility (light red to deep red bands on 1400 $\mathrm{veh} / \mathrm{hr}$ flow rate). Another finding of this analysis was that the closely spaced ADS vehicles with long platoons would generate more mobility improvements. Hence, the maximum mobility benefit was experienced in Case 33 and Case 49. Although the analysis concluded that closely spaced, long ADS platoons could attain higher mobility benefits, close proximity of ADS platoons and long chain of ADS vehicles in these platoon configurations would severely restrict merging vehicles from neighboring lanes, on-ramps, side roads, etc.

Analysis on platoon parameters at different traffic state revealed that, with other parameters being constant, increasing platoon length resulted in improved mobility gains. Similar investigation on inter-platoon headway presented that increase in inter-platoon headway would reduce traffic mobility if other two parameters remain constant at a specific traffic state. Analysis of intra-platoon headways coincides with the insights of inter-platoon headway analysis. Therefore, compactness of ADS vehicles would bring more mobility benefits in roadway sections with minimal conflict points (e.g., spans between on/off-ramps on freeways and sections between intersections in arterial). The notion of conflict points led to the next section of this study, examining the impact of ADS vehicles on traffic safety.
5.3. Impact on Safety. Although Cases 33 and 49 were found to be an obvious choice among 64 tested platoon configuration cases with respect to mobility enhancements, all aforementioned cases were examined again to identify the potential impact on traffic safety. Findings from ADS vehicles location and distribution influenced the simulation of safety improvements by placing a series of ADS vehicles at the front of traffic stream to obtain optimal benefits. Since no merging traffic was considered, the safety enhancements were examined as a measure of potentials to reduce rearend collision risks. Three safety surrogate measures were considered in this regard: time-to-collision (TTC), time exposed time-to-collision (TET), and time integrated timeto-collision (TIT).

TTC, TET, and TIT, introduced by Hayward, Minderhoud, and Bovy $[44,45]$, were widely used by traffic safety researchers. The time required for two successive vehicles in the same lane to hit if they maintain their current velocity is represented by TTC. Higher TTC would indicate safer traffic condition. TTC can be used to evaluate safety of a traffic environment, since lower TTC is indicative of potential dangerous situation [46]. Both TET and TIT are derived from TTC to measure safety improvements from macroscopic standpoint. Since TET is the summation of instances when TTC are lower than threshold value, the lower TET value is expected at safer traffic conditions. TET value was measured by (9) where TTC values for each vehicle at each time stamp $\left(T_{T C} C_{i, j}\right)$ were compared with the threshold TTC $\left(T T C^{*}\right)$ value to calculate TET value for each scenario. TIT measures the value of TTC lower than the threshold TTC. Similar to TET, a higher TIT value indicates higher safety concerns. The values of these parameters were measured using the following equations:

$$
\begin{aligned}
& \text { TTC }_{i, j}= \begin{cases}\frac{p_{i-1, j}-p_{i, j}-L}{v_{i, j}-v_{i-1, j}} & \text { if } v_{i, j}>v_{i-1, j} \\
\text { Inf } & \text { if } v_{i, j} \leq v_{i-1, j}\end{cases} \\
& T E T=\sum_{j=1}^{J} T E T_{j} \\
& \operatorname{TET}_{j}=\sum_{i=1}^{I} \delta_{j} \Delta j, \quad \delta_{j}= \begin{cases}1 & \forall 0<T T C_{i, j}<T T C^{*} \\
0 & \text { else }\end{cases} \\
& T I T=\sum_{j=1}^{J} T I T_{j} \\
& \operatorname{TIT}_{j}=\sum_{i=1}^{I}\left[\frac{1}{T T C_{i, j}}-\frac{1}{T T C^{*}}\right] . \Delta j \\
& \forall 0<T T C_{i, j}<T T C^{*}
\end{aligned}
$$

The threshold TTC values to measure TET and TIT were set as $2.5 \mathrm{sec}$, similar to standard perception reaction time. Resulting changes with regard to safety are displayed in Figure 4. Figure 4(a) presents total TET and average TIT values over the simulation period on base cases which 


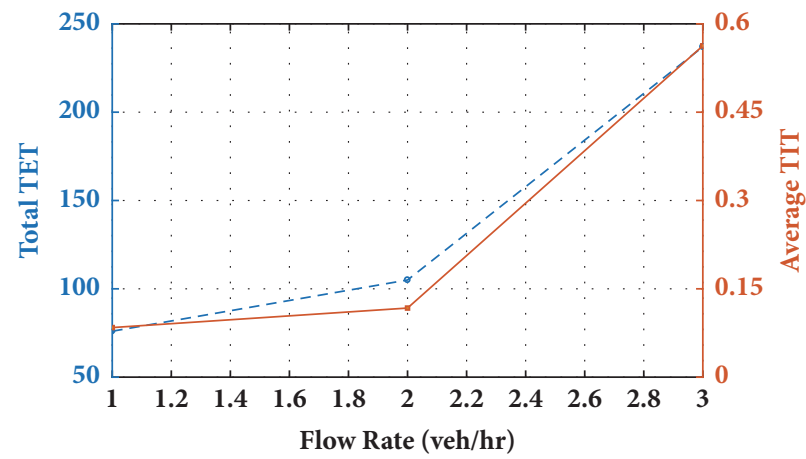

(a)
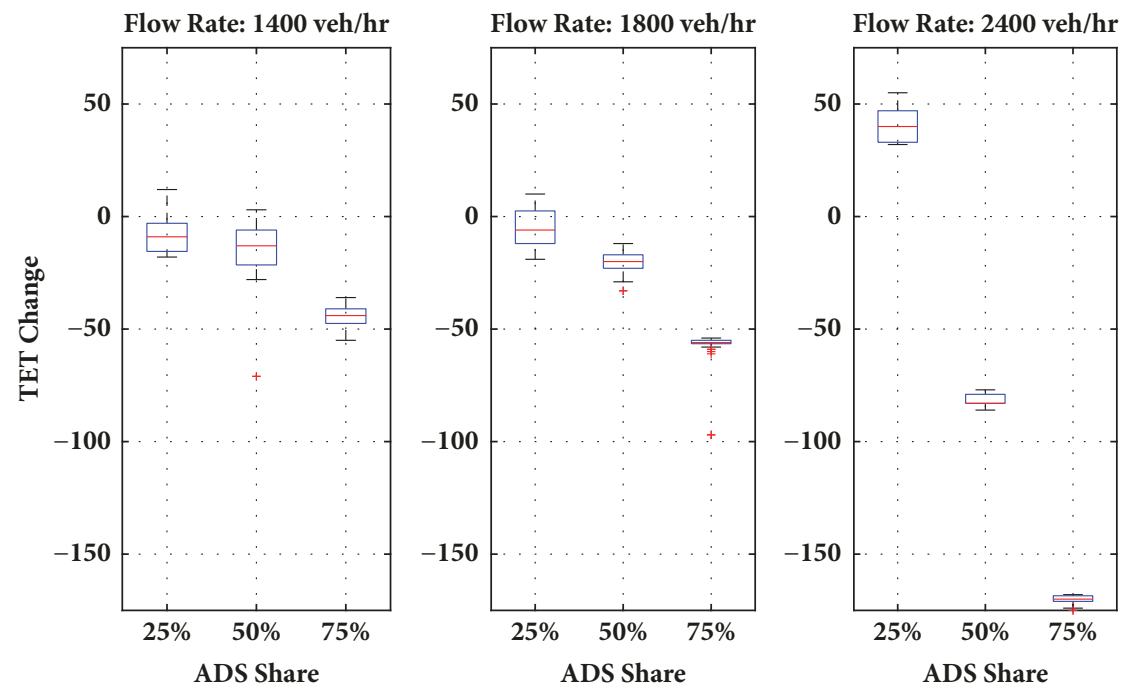

(b)

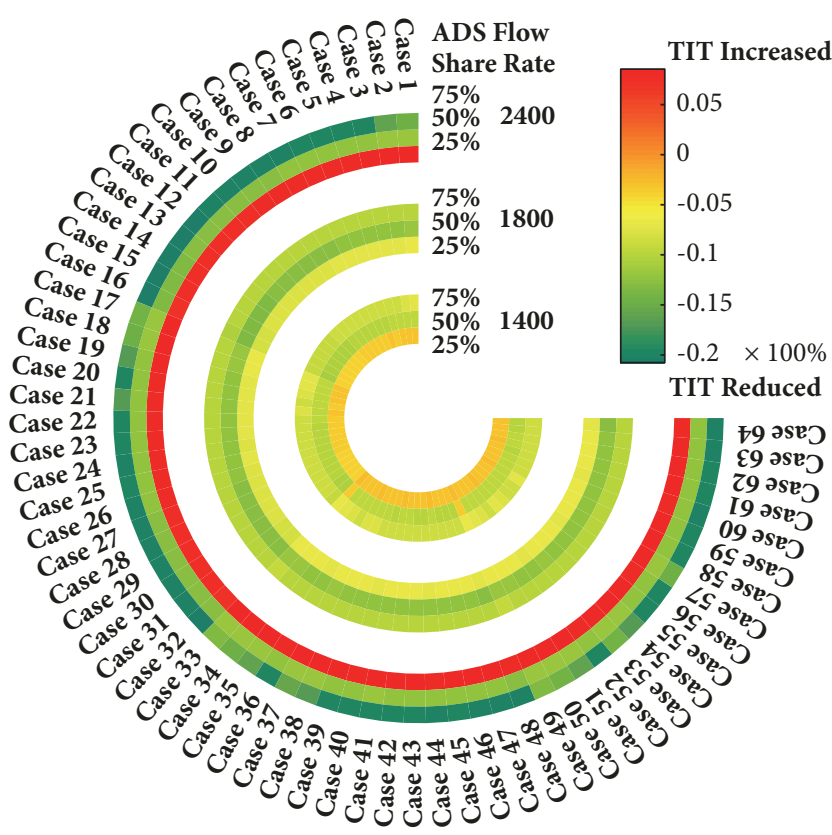

(c)

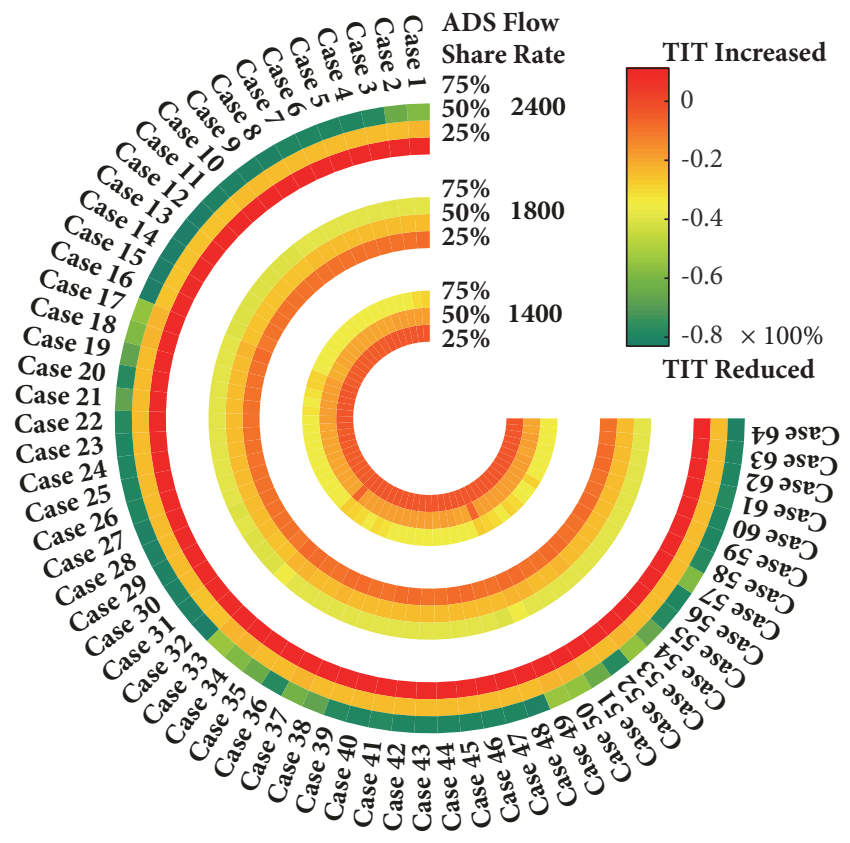

(d)

FIGURE 4: (a) Base-case safety parameter values at varying flow rates. (b) Changes in total TET, (c) variations of average TIT values considering MDS vehicles only, and (d) variations of average TIT values considering all vehicles, due to varying platoon configurations at different flow rates and ADS shares. 
were utilized to measure safety improvements gained with the introduction of ADS vehicles. Figure 4(b) displays the range of changes on total TET values at different traffic states with varying platoon structures. Increasing ADS shares showed a gradual decline of total TET values. The extent of declination was much higher in higher flow rates. However, an exception was observed at high flow rates and lower ADS shares (flow rate $=2400 \mathrm{veh} / \mathrm{hr}$, ADS share $=25 \%$ ) where total TET value increased from base traffic states. Therefore, it can be stated that higher ADS share is required to bring noticeable safety improvements with increasing flow rates. Figures 4(c) and 4(d) show analysis results of average TIT changes. As shown in earlier figure (Figure 3(a)), both factional circles revealed resulting improvements on average TIT parameters. Figure 4(c) shows resulting safety improvements of the vehicle stream for different platoon configurations, ADS shares, and flow rates by comparing with base average TIT values. This analysis considered average TIT values of MDS vehicles only in the traffic stream. Average TIT values of MDS vehicles in the CAV environment were compared with corresponding vehicles on base case for this analysis. The average TIT reduction of MDS vehicles was found to be within the range of $-20.76 \%-8.55 \%$. Additionally, higher safety gains were achieved with shorter platoons including ADS vehicles sparsely spaced.

On the other hand, Figure 4(d) shows the analysis by comparing average TIT values of all vehicles with base case. For this analysis, it was assumed that there was no collision risk for $\mathrm{ADS}$ vehicles (average TIT values $=0$ for $\mathrm{ADS}$ vehicles), irrespective of platoon configurations. Comparison between Figures 4(c) and 4(d) shows significantly higher improvements on average TIT values for all vehicles over MDS vehicles. The range of average reduction is much higher in Figure 4(d). Detailed analysis of safety enhancement for a specific traffic state provided further insights on the impact of platoon configurations. For instance, simulation results of $1800 \mathrm{veh} / \mathrm{hr}$ flow rate with $75 \%$ ADS share traffic state revealed that increasing ADS vehicles' stretch over the traffic stream resulted in greater safety benefits for the remaining vehicles. Hence, the maximum safety gain was attained from Case 16 (-10.23\% reduction on average TIT of MDS vehicles) for this specific traffic state. Although a similar pattern was observed for other traffic states, unexpectedly high safety concerns were experienced for some cases (dark red strip in Figure 4(c) for $2400 \mathrm{veh} / \mathrm{hr}$ with 25\% ADS share). Moreover, maximum safety gains on MDS vehicles were obtained on $50 \%$ ADS share at $1800 \mathrm{veh} / \mathrm{hr}$ flow. The findings from safety impact analysis have led us to conclude that increasing ADS vehicles with increasing flow rates would improve safety of all vehicles if ADS vehicles form short, sparse platoon in start of traffic stream. Although rear-end collision risk for MDS vehicles would proportionately reduce with increasing ADS share at comparatively high and low flow rate, this correlation between safety gain and ADS share did not hold true for flow rates near capacity level.

Exploring the evolution pattern of platoon parameters provided important insights into safety feature. While other parameters (i.e., inter-platoon headway and max. platoon length) remain the same, continuous increment of intra-platoon headway showed reduction on rear-end collision expectation. Inter-platoon headway followed similar pattern to intra-platoon headway. However, range of safety improvement in both parameters depends on maximum platoon length. Magnitude of safety gains was much higher at small platoons (i.e., max. platoon length $=3$ ) than big platoons (i.e., max. platoon length).

5.4. Impact on Environment. Environmental implications of proposed car-following mechanism were measured with respect to fuel consumption and emission reduction. While numerous models were available and utilized in the literature [47-49], the integrational simplicity of the VT-micro model [50-52] with car-following model persuaded us to implement this model. Output from car-following models can be directly used on the VT-micro model as input to estimate environmental impact due to vehicle dynamics which makes this model a perfect candidate for this analysis. According to the VT-micro model, the fuel consumption of or emission rate of $i^{\text {th }}$ vehicle at time step $j$ can be measured using the following equations:

$$
\ln \left(M_{i, j}\right)= \begin{cases}\sum_{l=0}^{3} \sum_{m=0}^{3} K_{l, m} \times v_{i, j}^{l} \times a_{i, j}^{m} & \text { if } a \geq 0 \\ \sum_{l=0}^{3} \sum_{m=0}^{3} K_{l, m}^{\prime} \times v_{i, j}^{l} \times a_{i, j}^{m} & \text { if } a<0\end{cases}
$$

where $M O E_{i, j}$ is measure of effectiveness with respect to fuel consumptions, $\mathrm{CO}_{2}$ emissions, and $\mathrm{NO}_{\mathrm{x}}$ emissions for vehicle $i$ at time $j$ and $K_{l, m}$ are regression coefficients for MOEs at powers $l$ and $m$. The values of regression coefficients are obtained from [50]. $v_{i, j}^{l}$ is velocity of vehicle $i$ at time $j$ with power $l . a_{i, j}^{m}$ is acceleration of vehicle $i$ at time $j$ with power $m$.

Analysis using the VT-micro model for the base case ( $0 \%$ ADS share) measured average fuel consumptions, $\mathrm{CO}_{2}$ emissions, and $\mathrm{NO}_{\mathrm{x}}$ emissions of the vehicles in simulated traffic stream at different flow rates, which is presented in Figure 5(a). Simulation results indicated that the lowest fuel consumption, $\mathrm{CO}_{2}$ emission, and $\mathrm{NO}_{\mathrm{x}}$ emission at base traffic state occurred at $1800 \mathrm{veh} / \mathrm{hr}$ flow rate. Therefore, low flow rates do not necessarily ensure low environmental impact. The transformation in environmental impact resulting from varying shares of ADS vehicles is demonstrated in Figures 5(b), 5(c), and 5(d). Gradual increments of ADS share showed a continuous reduction in fuel consumption. However, $\mathrm{CO}_{2}$ and $\mathrm{NO}_{\mathrm{x}}$ emissions for the traffic stream followed a different trend. As previous figures, the fractional circles displayed changes in average environmental parameters resulting from varying platoon structures and traffic states (i.e., flow rates and ADS shares). For a specific traffic state (i.e., flow rate and ADS share), the effect on environment demonstrated similar patterns to the impact on mobility. As an example, we can examine the platoon structures for $1800 \mathrm{veh} / \mathrm{hr}$ flow rate with $75 \%$ ADS share. The observations of this specific traffic state revealed that environmental benefits kept increasing with the gradual compaction of ADS vehicle in traffic stream. For instance, maximum reduction on fuel consumption was obtained for Case 33 and Case 49 (platoon configuration: 


\begin{tabular}{c|c|c|c|c}
\hline \multicolumn{5}{c}{ Base Case (0\% ADS Share) } \\
\hline Parameters & Unit & \multicolumn{3}{|c}{ Flow Rate (veh/hr) } \\
\cline { 3 - 5 } Fuel Consumption & $\begin{array}{c}\text { Litre/ } \\
100 \mathrm{~km}\end{array}$ & 8.57 & 8.52 & 8.66 \\
\hline $\mathrm{CO}_{2}$ Emission & $\begin{array}{c}\mathrm{Kg} / \\
100 \mathrm{~km}\end{array}$ & 19.71 & 19.64 & 19.9 \\
\hline $\mathrm{NO}_{\mathrm{x}}$ Emission & $\begin{array}{c}\mathrm{g} / \\
100 \mathrm{~km}\end{array}$ & 28.03 & 27.96 & 27.33 \\
\hline
\end{tabular}

(a)

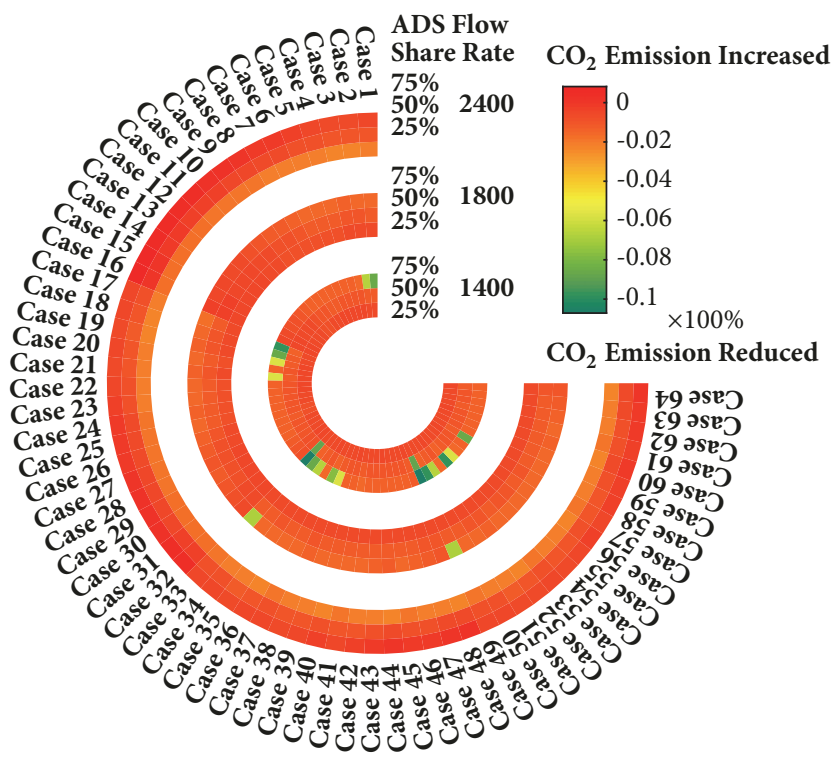

(c)

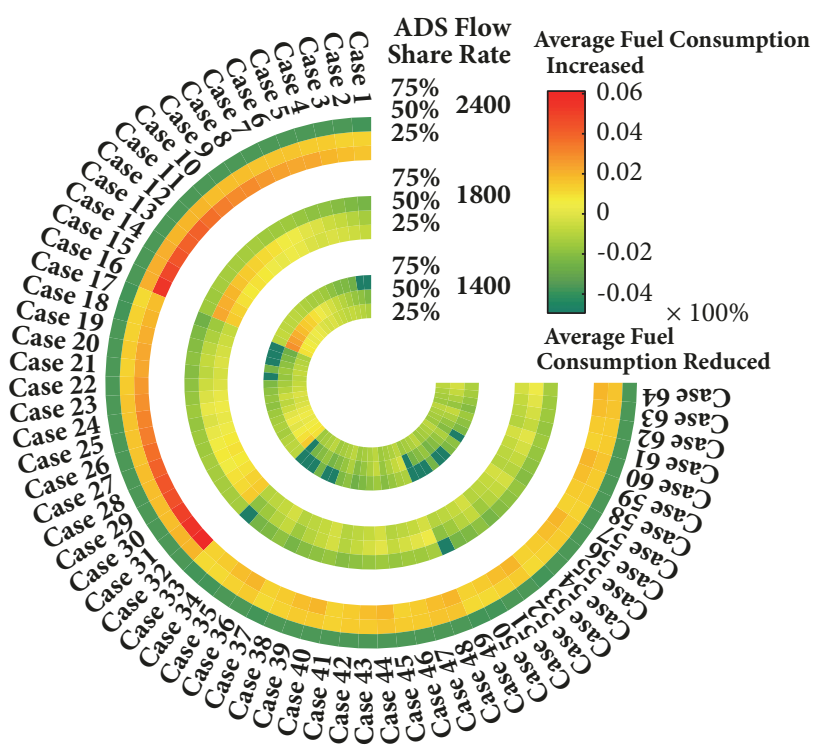

(b)

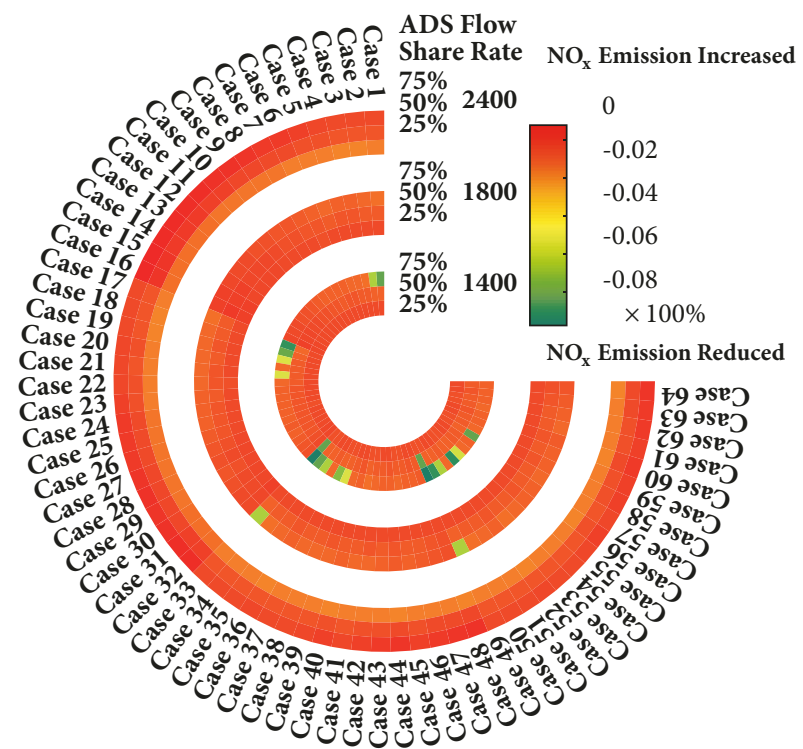

(d)

FIGURE 5: Observed variations of (a) environmental parameters at base case, (b) fuel consumption, (c) $\mathrm{CO}_{2}$ emission, and (d) $\mathrm{NO}_{\mathrm{x}}$ emission for varying platoon structures at different traffic state.

intra-platoon headway $=0.50 \mathrm{sec}$, inter-platoon headway $=2 \mathrm{sec}$, and max. platoon length $=5$ and 6 , respectively) which reduced average fuel consumption by $4.87 \%$ from the base case, whereas Cases 48 and 64 (platoon configuration: intra-platoon headway $=1.25 \mathrm{sec}$, inter-platoon headway $=$ $8 \mathrm{sec}$, and max. platoon length $=5$ and 6] reduced fuel consumption by $1.42 \%$ and $1.44 \%$, respectively. A similar pattern was observed for the other two parameters (i.e., $\mathrm{CO}_{2}$ emission and $\mathrm{NO}_{\mathrm{x}}$ emission) for this traffic state. Detailed analysis of the environmental impact identified a proportional relation between fuel consumption reduction and ADS share at all simulated traffic flow rates. However, the extent of improvements varied at different flow rates. As for $\mathrm{CO}_{2}$ and
$\mathrm{NO}_{\mathrm{x}}$ emission, the relation between emission reduction and ADS share was found to be proportionate at lower flow rates (i.e., 1400, $1800 \mathrm{veh} / \mathrm{hr}$ ). At high flow rate (i.e., $2400 \mathrm{veh} / \mathrm{hr}$ ), higher reduction was obtained at low ADS share. Out analysis of the environmental impact of ADS vehicles and formed platoons provided us with the insights of fuel consumption, $\mathrm{CO}_{2}$ emission, and $\mathrm{NO}_{\mathrm{x}}$ emission characteristics in order to make informed decision regarding platoon structures with an aim to attain optimal environmental benefits.

Close inspection of platoon parameters revealed similar inclinations to mobility. Unlike mobility gains, the degree of environmental gains was significantly higher at larger platoon sizes (i.e., max. platoon length $=5,6$ ) in comparison to smaller 
platoons (i.e., max. platoon length $=3,4$ ). Furthermore, the increments of intra and inter-platoon headway values showed steady declination of environmental benefits at a specific traffic state with other parameters being constant.

\section{Identification of Optimal Platoon Parameter Set}

An analysis of proposed car-following strategy delivered insights regarding mobility, safety, and environmental improvement potentials due to presence of ADS vehicles at mixed traffic conditions. One key finding of the analysis was that the expectation to obtain multiobjective improvements (i.e., mobility, safety, and environmental) from single platoon configuration was impractical. Since mobility and environmental developments maintained a reciprocal relationship with safety enhancements, a suboptimal platoon configuration could be determined to procure maximum gains from these three features. Another compelling outcome of prior analysis involved recognizing the fact that both traffic flow rates and ADS market shares impacted obtained benefits. Hence, achieving maximum mobility, safety, and environmental advantages from fixed suboptimal platoon configuration at different flow rates was unrealistic. To this end, it was necessary to present an approach that identified dynamic suboptimal platoon configurations for multiobjective decision-making purposes.

Influenced by Khondaker and Kattan [53], an analysis was performed to identify the suboptimal platoon configurations to maximize mobility, safety, and environmental gains generated by ADS vehicles. Collective influences from these three features were measured by placing different weights on them to get resulting variations on improvements (Figure 6(b)). Four sets of multiobjective functions were investigated to obtain suitable platoon structure. Sets for platoon variables were chosen from earlier analyses to identify suboptimal configurations.

The optimization of platoon variables for different multiobjective function identified each features' (i.e., mobility, safety, and environmental) individual and collective inclinations. To obtain clear and precise insights of these trends, the group of vehicles with $1800 \mathrm{veh} / \mathrm{hr}$ flow rate and $75 \%$ ADS market penetration is demonstrated in Figure 6. The improvements obtained due to ADS vehicles were scaled within [0 1] using extreme values from prior analysis of all the features (Figure 6(a)). For mobility improvements, the scenario scores were scaled within the above-mentioned range. Extreme average TIT values measured in safety impact analysis were applied to measure safety scores of different platoon configurations. Similarly, environmental score was calculated by assigning equal weights to three components of environmental impact (i.e., fuel consumption, $\mathrm{CO}_{2}$ emission, and $\mathrm{NO}_{\mathrm{x}}$ emission) while scaling them within the range of 0 and 1 . This action was performed due to variations of units in measures of effectiveness and to bring them in the same scale for optimization. Reviews of individual features identified a gradual reduction of mobility and environmental improvements with an increase of intra and inter-platoon headway. However, safety improvements showed opposite pattern. Figure 6(b) shows the results of a set of objective functions with predefined weight put on mobility, safety, and environmental aspect. The goal of this analysis is to obtain suboptimal platoon configurations for predefined objective sets and also to identify the objective function with maximum benefits from the assorted weight sets. Analysis of combined impacts identified that maximum benefits for all objective functions were achieved with the platoon configuration of intra-platoon headway $=0.50 \mathrm{sec}$, inter-platoon headway $=2$ sec, and maximum platoon length $=5 / 6$ vehicles. The objective of this analysis was to present an approach to identify suboptimal platoon configurations suitable for specific flow rates and ADS market share with specific motivation to assist in multiobjective decision-making.

\section{Conclusion and Future Extensions}

The objective of the study was to obtain rationalized insight on mixed traffic movements and evaluate the impact that ADS vehicles will supposedly have on traffic. While the potential of connectivity and automated controls is astounding, the extent of harnessing the benefits depends on discerning their influences on traffic. In this regard, we have proposed a naïve car-following mechanism for mixed traffic and analyzed their motion dynamics to determine the possible improvements. Initially, the location and distributions of ADS vehicles along the traffic stream were discovered to be moving forward with established framework to obtain the highest rewards. The mobility, safety, and environmental gains obtained from CAV traffic stream were examined for varying traffic flow, ADS market penetration, and platoon configurations with the intention of determining the limits of these potential improvements. The final stage of this study was the analysis to obtain optimal platoon configurations to achieve maximum collective improvements.

The findings of the research show that, to obtain maximum mobility benefits, close and compact platoons are favorable in roadway sections without side frictions. However, segments with on-ramps, off-ramps, side roads, etc. need to be researched in the future to account for side frictions and their consequences on collective mobility, safety, and environmental gains. Identifying suboptimal platoon configurations for varying flow rates and market shares of ADS vehicles will assist traffic operation authorities to propose traffic state responsive dynamic platoon structures. Utilizing these platoon configurations will make the best use of ADS vehicles on prevailing traffic conditions to obtain maximum gains. Future research based on this study will account for vehicles with conflicting movements (i.e., lane changing, merging traffic from on-ramps, diverging traffic towards off-ramp, etc.) and propose potential improvements.

\section{Data Availability}

The data used to support the findings of this study are available from the corresponding author upon request. 


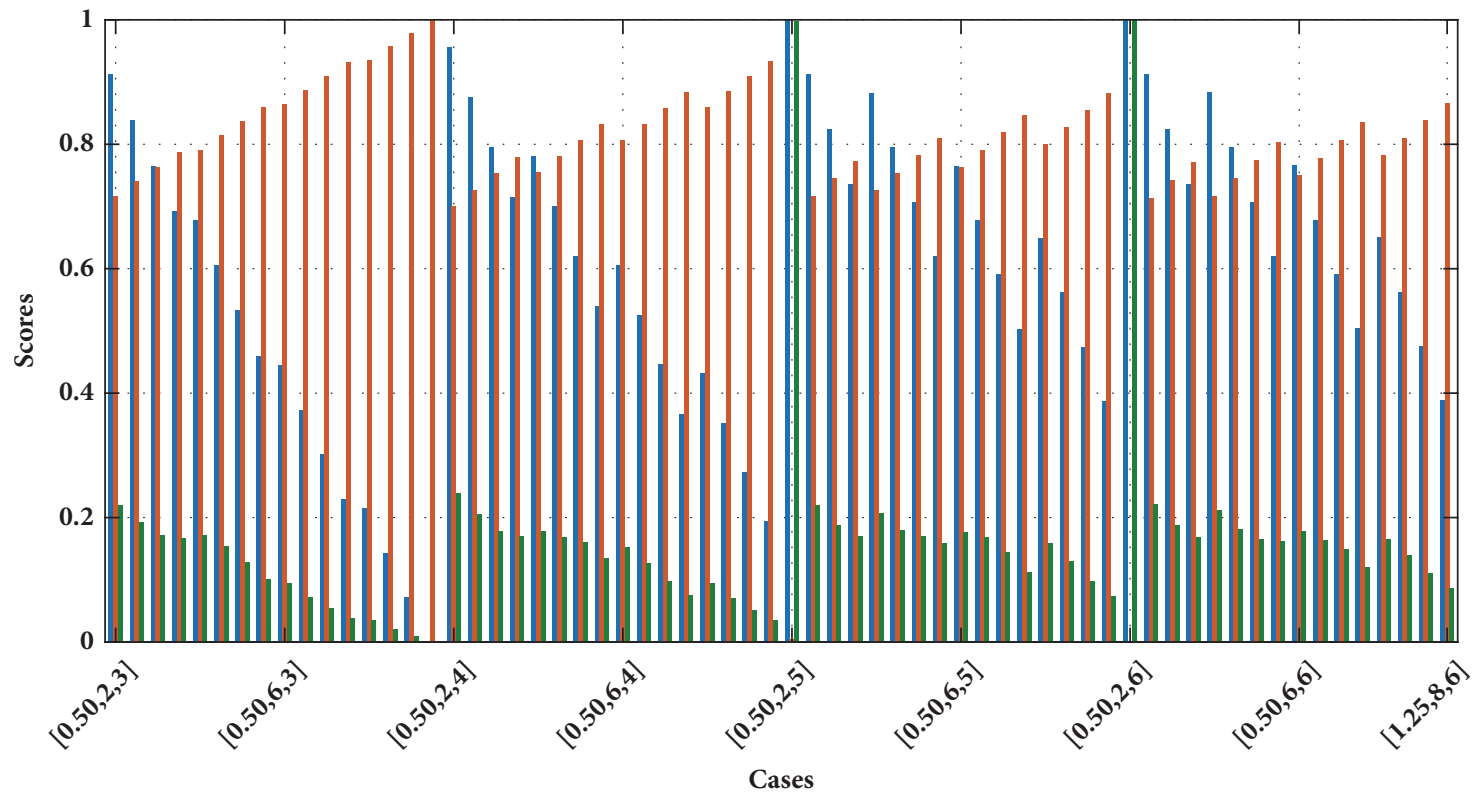

[Intra-platoon Headway, Inter-platoon Headway, Max. Platoon Length]

Mobility

Safety

Environment

(a)

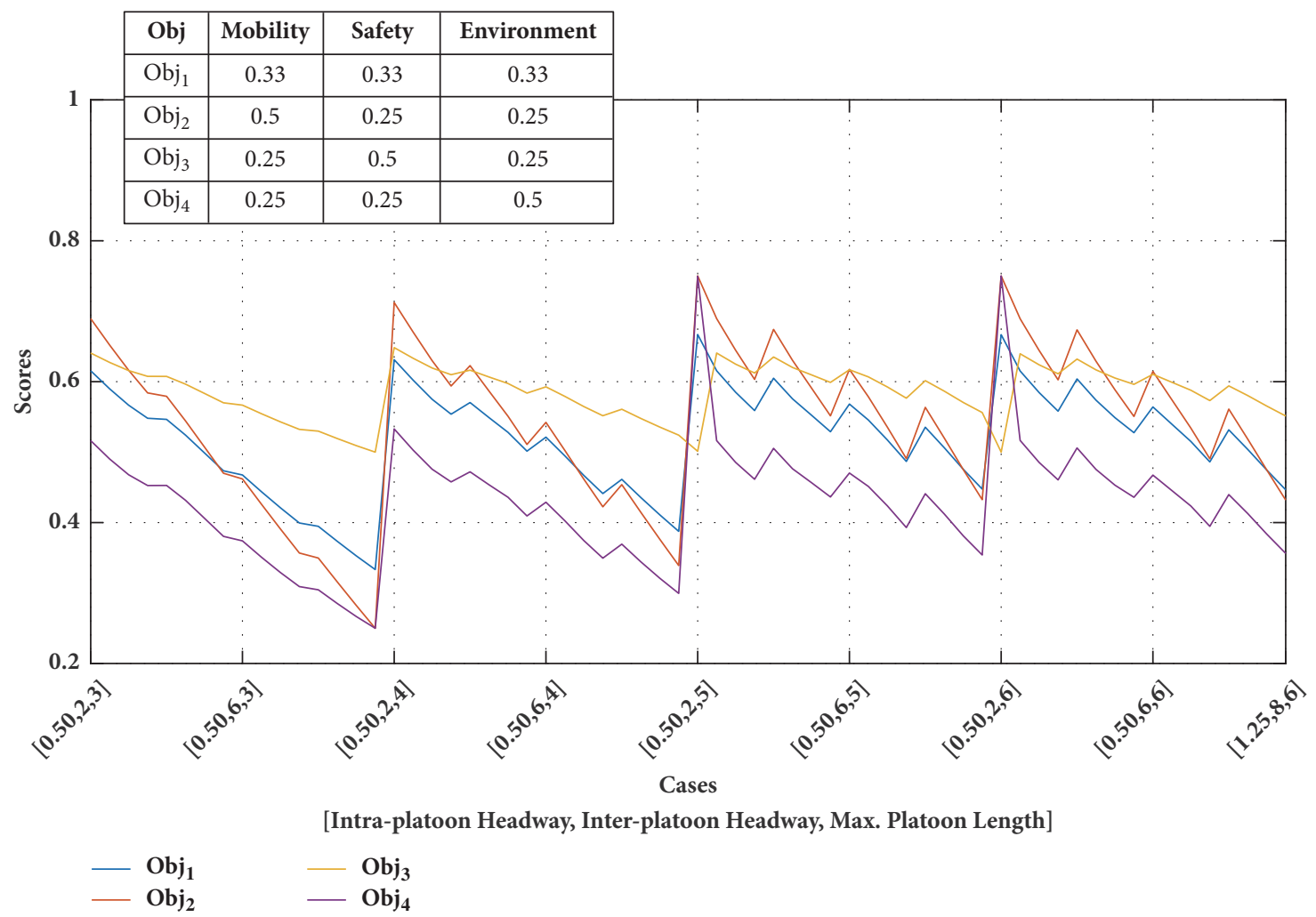

(b)

FIGURE 6: Observed variations of (a) individual features due to diverse platoon variables listed and (b) listed multiobjective function sets resulting from changing platoon variables. 


\section{Disclosure}

The contents of this paper reflect the views of the authors who are responsible for the facts and the accuracy of the data presented herein. The contents do not necessarily reflect the official views or policies of the City of Edmonton and Transport Canada. This paper does not constitute a standard, specification, or regulation.

\section{Conflicts of Interest}

The authors declare that they have no conflicts of interest.

\section{Acknowledgments}

This research work was jointly supported by the Natural Sciences and Engineering Research Council (NSERC) of Canada, City of Edmonton, and Transport Canada.

\section{References}

[1] S. Fakharian, "Evaluation of Cooperative Adaptive Cruise Control (CACC) Vehicles on Managed Lanes Utilizing Macroscopic and Mesoscopic Simulation," Transp. Res. Rec. J. Transp. Res. Board, vol. no, p. 16, 2016.

[2] A. Ghiasi, O. Hussain, Z. Qian, and X. P. Li, "A mixed traffic capacity analysis and lane management model for connected automated vehicles: A Maekov chain method," Transportation Research Part B: Methodological, vol. 106, pp. 266-292, 2017.

[3] Y. Liu, J. Guo, J. Taplin, and Y. Wang, "Characteristic Analysis of Mixed Traffic Flow of Regular and Autonomous Vehicles Using Cellular Automata," Journal of Advanced Transportation, vol. 2017, Article ID 8142074, 10 pages, 2017.

[4] A. Talebpour and H. S. Mahmassani, "Influence of connected and autonomous vehicles on traffic flow stability and throughput," Transportation Research Part C: Emerging Technologies, vol. 71, pp. 143-163, 2016.

[5] N. Bekiaris-Liberis, C. Roncoli, and M. Papageorgiou, "Highway traffic state estimation per lane in the presence of connected vehicles," Transportation Research Part B: Methodological, vol. 106, pp. 1-28, 2017.

[6] D. Chen, S. Ahn, M. Chitturi, and D. A. Noyce, "Towards vehicle automation: Roadway capacity formulation for traffic mixed with regular and automated vehicles," Transportation Research Part B: Methodological, vol. 100, pp. 196-221, 2017.

[7] R. E. Chandler, R. Herman, and E. W. Montroll, "Traffic dynamics: studies in car following," Operations Research, vol. 6, pp. 165-184, 1958.

[8] W. Helly, "Simulation of bottlenecks in single-lane traffic flow," in Theory of traffic flow, pp. 207-238, Elsevier, Amsterdam, 1961.

[9] M. Bando, K. Hasebe, A. Nakayama, A. Shibata, and Y. Sugiyama, "Dynamical model of traffic congestion and numerical simulation," Physical Review E: Statistical, Nonlinear, and Soft Matter Physics, vol. 51, no. 2, pp. 1035-1042, 1995.

[10] M. Treiber, A. Hennecke, and D. Helbing, "Congested traffic states in empirical observations and microscopic simulations," Physical Review E: Statistical, Nonlinear, and Soft Matter Physics, vol. 62 , no. 2, pp. 1805-1824, 2000.

[11] P. G. Gipps, "A behavioural car-following model for computer simulation," Transportation Research Part B: Methodological, vol. 15, no. 2, pp. 105-111, 1981.
[12] L. Evans and R. Rothery, Experimental measurement of perceptual thresholds in car following. Highway Research Board, Washington District of Columbia, United States, 1973.

[13] L. C. Davis, "Optimality and oscillations near the edge of stability in the dynamics of autonomous vehicle platoons," Physica A: Statistical Mechanics and its Applications, vol. 392, no. 17, pp. 3755-3764, 2013.

[14] P. Y. Li and A. Shrivastava, "Traffic flow stability induced by constant time headway policy for adaptive cruise control vehicles," Transportation Research Part C: Emerging Technologies, vol. 10, no. 4, pp. 275-301, 2002.

[15] G. Orosz, J. Moehlis, and F. Bullo, "Delayed car-following dynamics for human and robotic drivers," in Proceedings of the ASME 2011 International Design Engineering Technical Conferences and Computers and Information in Engineering Conference, IDETC/CIE 2011, pp. 529-538, USA, August 2011.

[16] L. Xiao and F. Gao, "Practical string stability of platoon of adaptive cruise control vehicles," IEEE Transactions on Intelligent Transportation Systems, vol. 12, no. 4, pp. 1184-1194, 2011.

[17] S. G. Hu, H. Y. Wen, L. Xu, and H. Fu, "Stability of platoon of adaptive cruise control vehicles with time delay," Transportation Letters, pp. 1-10, 2017.

[18] Z. Wang, G. Wu, and M. Barth, "Developing a distributed consensus-based Cooperative Adaptive Cruise Control (CACC) system," J. Adv. Transp, vol. 2017, 2017.

[19] M. Fountoulakis, N. Bekiaris-Liberis, C. Roncoli, I. Papamichail, and M. Papageorgiou, "Highway traffic state estimation with mixed connected and conventional vehicles: Microscopic simulation-based testing," Transportation Research Part C: Emerging Technologies, vol. 78, pp. 13-33, 2017.

[20] Q. Xin, N. Yang, R. Fu, S. Yu, and Z. Shi, "Impacts analysis of car following models considering variable vehicular gap policies," Physica A: Statistical Mechanics and its Applications, vol. 501, pp. 338-355, 2018.

[21] J. Sun, Z. Zheng, and J. Sun, "Stability analysis methods and their applicability to car-following models in conventional and connected environments," Transportation Research Part B: Methodological, vol. 109, pp. 212-237, 2018.

[22] B. Van Arem, C. J. G. Van Driel, and R. Visser, "The impact of cooperative adaptive cruise control on traffic-flow characteristics," IEEE Transactions on Intelligent Transportation Systems, vol. 7, no. 4, pp. 429-436, 2006.

[23] G. N. Bifulco, L. Pariota, F. Simonelli, and R. D. Pace, "Development and testing of a fully adaptive cruise control system," Transportation Research Part C: Emerging Technologies, vol. 29, pp. 156-170, 2013.

[24] J. Yi and R. Horowitz, "Macroscopic traffic flow propagation stability for adaptive cruise controlled vehicles," Transportation Research Part C: Emerging Technologies, vol. 14, no. 2, pp. 81-95, 2006.

[25] I. A. Ntousakis, I. K. Nikolos, and M. Papageorgiou, "On Microscopic Modelling of Adaptive Cruise Control Systems," Transportation Research Procedia, vol. 6, pp. 111-127, 2015.

[26] A. I. Delis, I. K. Nikolos, and M. Papageorgiou, "Macroscopic traffic flow modeling with adaptive cruise control: development and numerical solution," Computers \& Mathematics with Applications, vol. 70, no. 8, pp. 1921-1947, 2015.

[27] L. Ye and T. Yamamoto, "Modeling connected and autonomous vehicles in heterogeneous traffic flow," Physica A: Statistical Mechanics and its Applications, vol. 490, pp. 269-277, 2018. 
[28] W.-X. Zhu and H. M. Zhang, "Analysis of mixed traffic flow with human-driving and autonomous cars based on car-following model," Physica A: Statistical Mechanics and its Applications, vol. 496, pp. 274-285, 2018.

[29] H. Yeo, A. Skabardonis, J. Halkias, J. Colyar, and V. Alexiadis, "Oversaturated freeway flow algorithm for use in Next Generation Simulation," Transportation Research Record, no. 2088, pp. 68-79, 2008.

[30] N. Chen, M. Wang, T. Alkim, and B. van Arem, "A Robust Longitudinal Control Strategy of Platoons under Model Uncertainties and Time Delays," Journal of Advanced Transportation, vol. 2018, Article ID 9852721, 13 pages, 2018.

[31] N. Bekiaris-Liberis, C. Roncoli, and M. Papageorgiou, "Highway traffic state estimation with mixed connected and conventional vehicles," IEEE Transactions on Intelligent Transportation Systems, vol. 17, no. 12, pp. 3484-3497, 2016.

[32] V. A. C. van den Berg and E. T. Verhoef, "Autonomous cars and dynamic bottleneck congestion: the effects on capacity, value of time and preference heterogeneity," Transportation Research Part B: Methodological, vol. 94, pp. 43-60, 2016.

[33] P. Tientrakool, Y.-C. Ho, and N. F. Maxemchuk, "Highway capacity benefits from using vehicle-to-vehicle communication and sensors for collision avoidance," in Proceedings of the IEEE 74th Vehicular Technology Conference, VTC Fall, The Hilton San Francisco Union Square, San Francisco, USA, September 2011.

[34] J. Vander Werf, S. Shladover, M. Miller, and N. Kourjanskaia, "Effects of Adaptive Cruise Control Systems on Highway Traffic Flow Capacity," Transportation Research Record, vol. 1800, pp. 78-84, 2002.

[35] D. Ni, J. Li, S. Andrews, and H. Wang, "Preliminary estimate of highway capacity benefit attainable with IntelliDrive technologies," in Proceedings of the 13th International IEEE Conference on Intelligent Transportation Systems, ITSC 2010, pp. 819-824, Portugal, September 2010.

[36] T.-H. Chang and I.-S. Lai, "Analysis of characteristics of mixed traffic flow of autopilot vehicles and manual vehicles," Transportation Research Part C: Emerging Technologies, vol. 5, no. 6, pp. 333-348, 1997.

[37] P. Fernandes and U. Nunes, "Platooning with IVC-enabled autonomous vehicles: Strategies to mitigate communication delays, improve safety and traffic flow," IEEE Transactions on Intelligent Transportation Systems, vol. 13, no. 1, pp. 91-106, 2012.

[38] N. H. T. S. Administration, "FMVSS No. 150 Vehicle-To-Vehicle Communication Technology For Light Vehicles," Off. Regul. Anal. Eval. Natl. Cent. Stat. Anal., no. 150, 2016.

[39] Y. Li, H. Wang, W. Wang, L. Xing, S. Liu, and X. Wei, "Evaluation of the impacts of cooperative adaptive cruise control on reducing rear-end collision risks on freeways," Accident Analysis \& Prevention, vol. 98, pp. 87-95, 2017.

[40] M. S. Rahman and M. Abdel-Aty, "Longitudinal safety evaluation of connected vehicles' platooning on expressways," Accident Analysis \& Prevention, vol. 117, pp. 381-391, 2018.

[41] M. Zabat, N. Stabile, S. Farascaroli, and F. Browand, "The Aerodynamic Performance Of Platoons: A Final Report," Calif. Partners Adv. Transit Highw, 1995.

[42] Z. Wang, G. Wu, P. Hao, K. Boriboonsomsin, and M. Barth, "Developing a platoon-wide Eco-Cooperative Adaptive Cruise Control (CACC) system," in Proceedings of the 28th IEEE Intelligent Vehicles Symposium, IV 2017, pp. 1256-1261, USA, June 2017.

[43] M. Mamouei, I. Kaparias, and G. Halikias, "A framework for user- and system-oriented optimisation of fuel efficiency and traffic flow in Adaptive Cruise Control," Transportation Research Part C: Emerging Technologies, vol. 92, pp. 27-41, 2018.

[44] J. C. Hayward, "Near-Miss Determination Through," Highw. Res. Board, pp. 24-35, 1971.

[45] M. M. Minderhoud and P. H. L. Bovy, "Extended time-tocollision measures for road traffic safety assessment," Accident Analysis \& Prevention, vol. 33, no. 1, pp. 89-97, 2001.

[46] K. Vogel, "A comparison of headway and time to collision as safety indicators," Accident Analysis \& Prevention, vol. 35, no. 3, pp. 427-433, 2003.

[47] M. Barth et al., "Development of a Comprehensive Modal Emissions Model," Natl. Coop. Highw. Res. Progr, 2000.

[48] J. Koupal, H. Michaels, M. Cumberworth, C. Bailey, and D. Brzezinski, "EPAs plan for MOVES: a comprehensive mobile source emissions model," in Proceedings of the 12th CRC OnRoad Veh. Emiss. Work, San Diego, CA.

[49] S. Hausberger, J. Rodler, P. Sturm, and M. Rexeis, "Emission factors for heavy-duty vehicles and validation by tunnel measurements," Atmospheric Environment, vol. 37, no. 37, pp. 52375245, 2003.

[50] K. Ahn, Microscopic Fuel Consumption and Emission Modeling, Virginia Tech University, 1998.

[51] K. Ahn, H. Rakha, A. Trani, and M. van Aerde, "Estimating vehicle fuel consumption and emissions based on instantaneous speed and acceleration levels," Journal of Transportation Engineering, vol. 128, no. 2, pp. 182-190, 2002.

[52] T.-Q. Tang, Z.-Y. Yi, and Q.-F. Lin, "Effects of signal light on the fuel consumption and emissions under car-following model," Physica A: Statistical Mechanics and its Applications, vol. 469, pp. 200-205, 2017.

[53] B. Khondaker and L. Kattan, "Variable speed limit: a microscopic analysis in a connected vehicle environment," Transportation Research Part C: Emerging Technologies, vol. 58, pp. 146-159, 2015. 


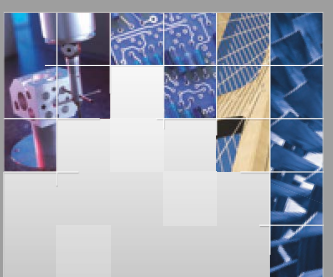

\section{Enfincering}
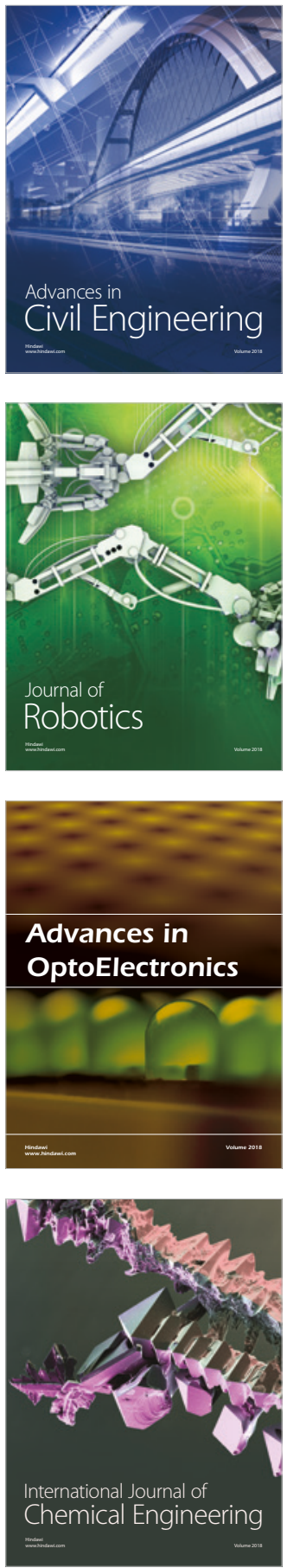

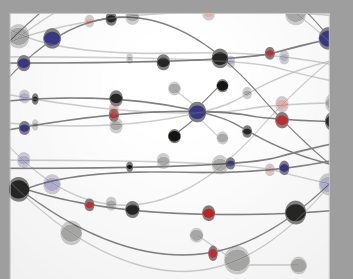

\section{Rotating \\ Machinery}

The Scientific World Journal

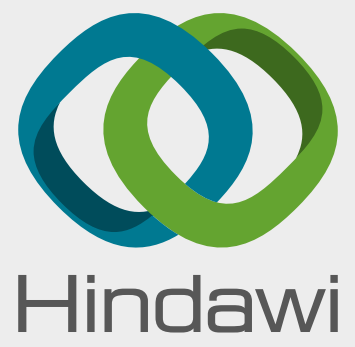

Submit your manuscripts at

www.hindawi.com
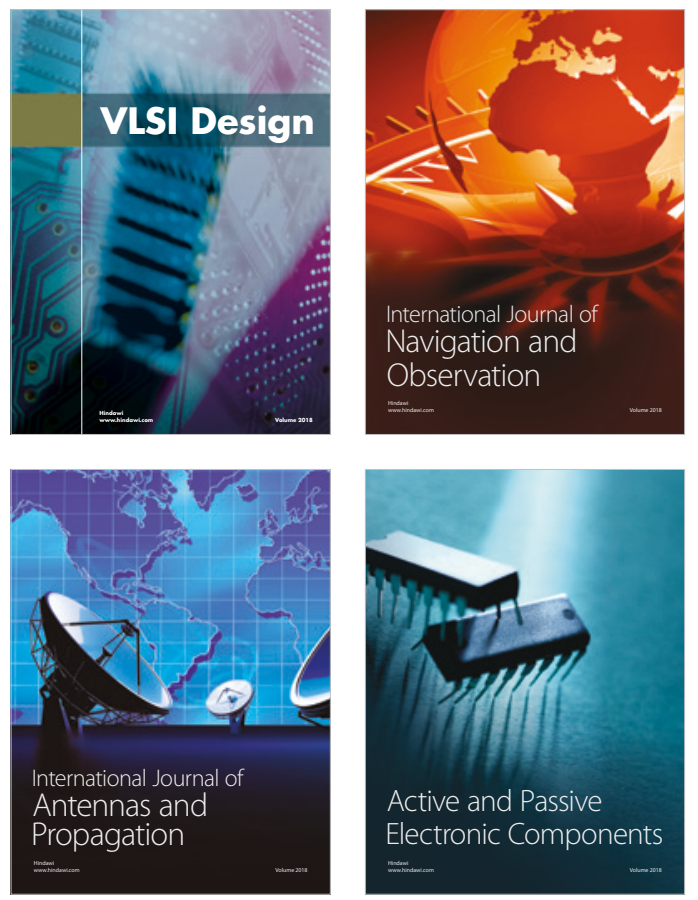
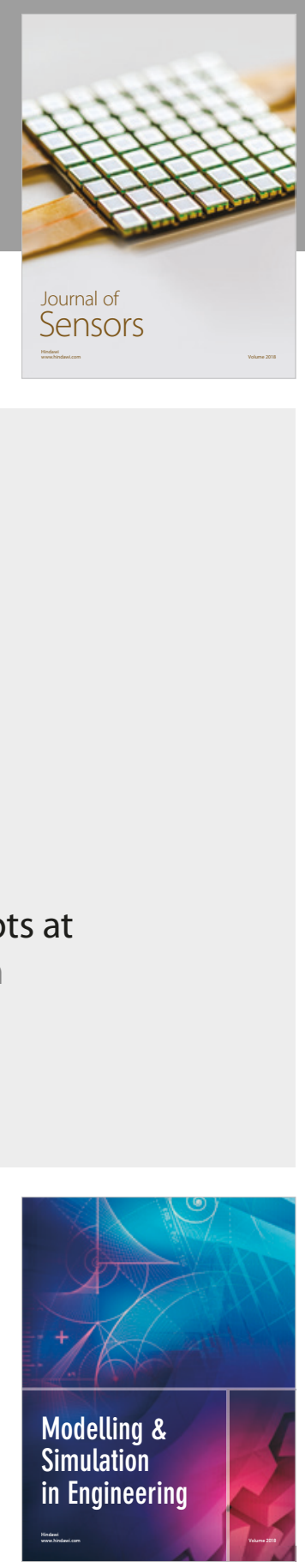

\section{Advances \\ Multimedia}
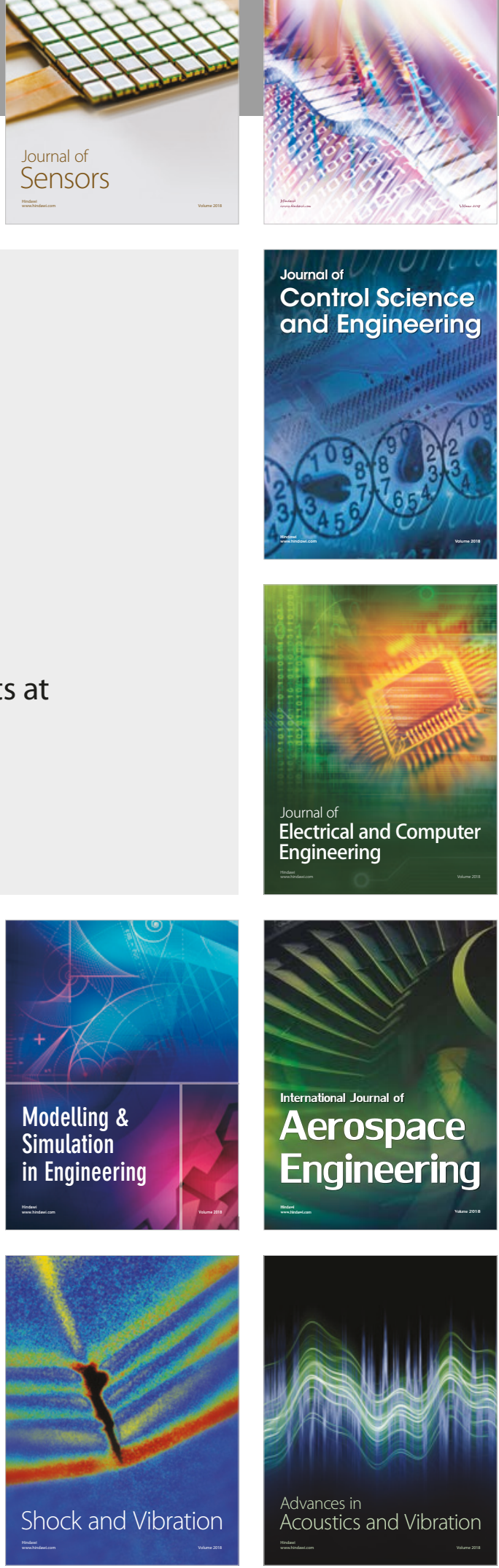